\title{
8-1998
}

\section{Extracting Classical Trajectories from Atomic Spectra}

M. R. Haggerty

William \& Mary

Neal Spellmeyer

Daniel Kleppner

John B. Delos

William \& Mary, jbdelos@wm.edu

Follow this and additional works at: https://scholarworks.wm.edu/aspubs

Part of the Physics Commons

\section{Recommended Citation}

Haggerty, M. R.; Spellmeyer, Neal; Kleppner, Daniel; and Delos, John B., Extracting Classical Trajectories from Atomic Spectra (1998). Physical Review Letters, 81(8), 1592-1595.

https://doi.org/10.1103/PhysRevLett.81.1592

This Article is brought to you for free and open access by the Arts and Sciences at W\&M ScholarWorks. It has been accepted for inclusion in Arts \& Sciences Articles by an authorized administrator of W\&M ScholarWorks. For more information, please contact scholarworks@wm.edu. 


\title{
Extracting Classical Trajectories from Atomic Spectra
}

\author{
M. R. Haggerty, ${ }^{1, *}$ Neal Spellmeyer, ${ }^{2}$ Daniel Kleppner, ${ }^{2}$ and J. B. Delos ${ }^{1}$ \\ ${ }^{1}$ Physics Department, College of William and Mary, Williamsburg, Virginia 23187 \\ ${ }^{2}$ Research Laboratory of Electronics, George R. Harrison Spectroscopy Laboratory, and Department of Physics, \\ Massachusetts Institute of Technology, 77 Massachusetts Avenue, Cambridge, Massachusetts 02139
}

(Received 18 December 1997)

\begin{abstract}
We describe how to reconstruct individual classical trajectories from spectroscopic data. The ac dipole moment of a trajectory can be found from the effect of an oscillating field on the spectrum. The inverse Fourier transform of such data yields the component of the electron trajectory along the direction of the oscillating field. We demonstrate the method by experimentally extracting $z(t)$ for two electron trajectories that influence the Stark spectrum of Rydberg lithium. Within the experimental resolution, the reconstructed orbits agree well with classical predictions. [S0031-9007(98)06988-9]
\end{abstract}

PACS numbers: $32.60 .+\mathrm{i}, 03.65 . \mathrm{Sq}, 05.45 .+\mathrm{b}$

The classical behavior of a dynamical system is expected to be derivable from its underlying quantum structure, and new methods of connecting classical and quantum approaches continue to be developed. Periodic orbit theory [1] and its variants allow one to learn about the actions and stabilities of classical orbits from a system's quantum density of states (though it is typically used the other way around). There are only a few methods that can be used to find the trajectories themselves their position as a function of time - and the methods are either indirect [2] or require knowledge of the quantum wave functions in addition to the spectrum $[3,4]$. We present here the results of a new study in which semiclassical methods are used to reconstruct a trajectory from experimental spectroscopic data.

When we speak of the "classical trajectory of an electron," we mean, of course, the path the electron would follow if it obeyed the laws of classical mechanics. In quantum mechanics an electron is not a localized object moving along a path. Nevertheless, a classical path is significant even in the quantum world - in semiclassical theories we use classical paths to construct wave functions and spectra. We demonstrate here that the process is invertible: under appropriate conditions such classical paths can be reconstructed from observed quantum spectra.

Rydberg atoms in external fields are an excellent laboratory for studying semiclassical methods experimentally. Their spectra can be interpreted with a variation of the periodic orbit theory known as closed orbit theory $[5,6]$. Closed orbit theory relates fluctuations in the atomic photoabsorption spectrum to the system's classical closed orbits (orbits that begin and end at the nucleus). A spectrum taken under conditions obeying classical scaling laws can be Fourier transformed to yield a "recurrence spectrum," in which each closed orbit appears as a peak in a plot of intensity vs action [7]. This procedure establishes the existence and action of the closed orbits, and provides some information about their stabilities and initial directions. From the change in peak positions when experimental pa- rameters are changed, it is also possible to learn about the periods and average electric dipole moments of the orbits [8]. However, the orbits themselves (the electron position as a function of time) have hitherto been experimentally inaccessible.

The idea that the shape of an orbit could be deduced from spectroscopic data arose from a study of recurrence spectra of Rydberg atoms in a static electric field, perturbed by an additional weak time-dependent electric field [9]. The oscillating field was observed to reduce the strengths of recurrences systematically. The effect was explained by generalizing closed orbit theory to timedependent systems. Most intriguingly, the pattern of the weakening depends on the Fourier transform of the classical orbits of the electron in the static system. But suppose the static part of the Hamiltonian (e.g., the configuration of electric and/or magnetic fields) was not known. Would it be possible to use oscillating field experiments to measure the Fourier transform of the motion for a range of frequencies, take the inverse Fourier transform, and thereby learn about the shape of the orbit?

We present here the results of a new study which shows that this is indeed possible. By doing spectroscopy in an oscillating field, we gain new information that allows us to reconstruct a trajectory directly-without measuring the wave function and without relying on detailed knowledge of the static Hamiltonian. We describe this new method and use it to reconstruct two electron orbits important to the Stark spectrum of lithium. Within the limits of the experiment, the measured orbits are in excellent agreement with the orbits predicted by classical simulation.

We use cw laser spectroscopy to study the Rydberg spectrum of lithium in a constant electric field $\mathbf{F}=F \hat{\mathbf{z}}$, perturbed with a weak oscillating field $\mathbf{F}_{1}=F_{1} \hat{\mathbf{z}} \cos (\omega t)$. The experimental setup is similar to that used in an earlier study of recurrence spectra in a static field [10]. The oscillating field is coupled through a static field plate, and its strength is calibrated by measuring the sideband structure on a low-lying Rydberg state. While 
the atoms are in the combined fields, we measure the laser photoexcitation rate from the $3 s$ state to final states corresponding to the principal quantum numbers around $n=125$ and $m=0$.

The system can be described by the Hamiltonian for hydrogen because the large-scale structure important to these experiments is unaffected by the lithium core electrons [10]. The Hamiltonian obeys a classical scaling law and can be written

$$
\tilde{H}=\frac{\tilde{p}^{2}}{2}-\frac{1}{\tilde{r}}+\tilde{z}[1+\tilde{f} \cos (\tilde{\omega} \tilde{t})]=F^{-1 / 2} E(t),
$$

where the tildes denote scaled quantities: $\tilde{r} \equiv F^{1 / 2} r$, $\tilde{p} \equiv F^{-1 / 4} p$, and $\tilde{t} \equiv F^{3 / 4} t$ [9]. Because of the scaling law, the unperturbed classical dynamics depends only on the scaled energy $\epsilon \equiv E_{0} F^{-1 / 2}$, where $E_{0}$ is the initial energy of the electron, measured relative to the field-free ionization threshold. The classical dynamics of the perturbed system depends on $\epsilon$ and also on the scaled parameters $\tilde{f} \equiv F_{1} / F$ and $\tilde{\omega} \equiv \omega F^{-3 / 4}$ which characterize the oscillating field.

We measure scaled spectra of this system by recording the photoabsorption spectrum as a function of $w \equiv F^{-1 / 4}$ while the laser energy, the static and rf field amplitudes, and the $\mathrm{rf}$ frequency are varied simultaneously so as to maintain $\epsilon, \tilde{f}$, and $\tilde{\omega}$ constant. The magnitude squared of the Fourier transform of a scaled spectrum with respect to $w$ is called a recurrence spectrum. Such a spectrum exhibits a peak at the scaled action $\tilde{S}_{k} \equiv F^{1 / 4} S_{k}$ of each classical closed orbit $k$ of the electron. Examples of recurrence spectra are shown in Fig. 1.

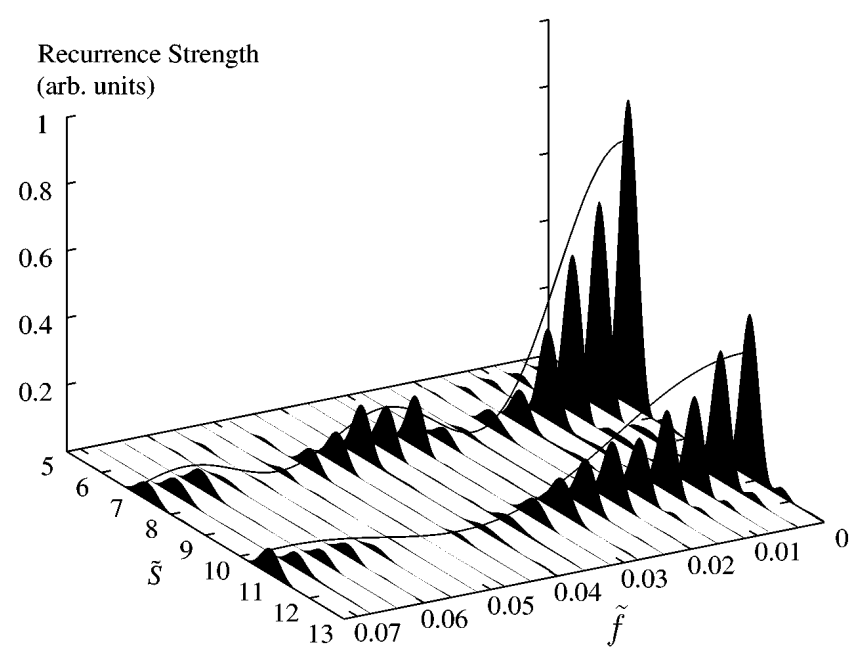

FIG. 1. A series of experimental recurrence spectra taken at $\epsilon=-2.05$ and $\tilde{\omega}=1.4$, with 19 different oscillating electric field strengths. The recurrences at $\tilde{S}=7.23$ correspond to the $2 / 3$ orbit, those at $\tilde{S}=10.68$ to the $3 / 4$ orbit. The lines are fits of the data to Eq. (2). The fits yield $\tilde{T}_{2 / 3}\left|\tilde{Z}_{2 / 3}\right|=0.417$ and $\tilde{T}_{3 / 4}\left|\tilde{Z}_{3 / 4}\right|=0.221$ (classical calculation gives 0.437 and 0.219 , respectively). The data were recorded from $248.8 \leq$ $w \leq 260.6$.
References $[9,11]$ investigate the effect of an oscillating field on a recurrence spectrum by considering how the oscillating field perturbs the classical orbits of the static system. Based on general semiclassical arguments, it can be shown that the recurrence spectrum of a static system perturbed by a weak oscillating field is similar to that of the static system alone, except that the recurrence strength (i.e., the height of the peak) associated with orbit $k$ is reduced by the factor

$$
a_{k}\left(F_{1}\right) \equiv J_{0}^{2}\left[F_{1} T_{k}\left|Z_{k}(\omega)\right| / \hbar\right] .
$$

$T_{k}$ is the period of the unperturbed orbit $k$, and $Z_{k}(\omega)$ is its complex ac dipole moment. The combination $T_{k} Z_{k}$ is given by

$$
T_{k} Z_{k}(\omega) \equiv \int_{-T_{k} / 2}^{T_{k} / 2} z_{k}(\tau) e^{-i \omega \tau} d \tau
$$

where $z_{k}(\tau)$ describes the $z$ motion of the electron along the unperturbed closed orbit as a function of time, leaving the atom at time $-T_{k} / 2$ and returning at time $T_{k} / 2$. (The $z$ motion is singled out by the polarization of the oscillating field.) Note that Eq. (2) applies even when the frequency of the rf field is comparable to or exceeds the frequencies of the classical orbits.

For the present case these formulas can be written in scaled variables, and the argument of the Bessel function becomes $\left[\tilde{f} \tilde{T}_{k}\left|\tilde{Z}_{k}(\tilde{\omega})\right| \bar{w}\right]$, where $\bar{w}$ is the average value of $F^{-1 / 4}$. We determine $\tilde{z}_{k}(\tau)$ from experimental data with the following procedure: (i) Measure recurrence spectra at a series of increasing values of $\tilde{f}$, at a fixed frequency $\tilde{\omega}$ (see Fig. 1). (ii) Select the recurrence peak corresponding to the classical orbit of interest, measure its height as a function of $\tilde{f}$, and from these results obtain experimental values of $a_{k}(\tilde{f})$ at that frequency. (iii) Obtain $\tilde{T}_{k}\left|\tilde{Z}_{k}(\tilde{\omega})\right|$ by fitting these data to the scaled version of Eq. (2). (iv) Repeat the entire process for successive values of $\tilde{\omega}$. (v) Insert the missing complex phase information to derive $\tilde{T}_{k} \tilde{Z}_{k}(\tilde{\omega})$ from its modulus (this step is explained below). (vi) Fourier invert Eq. (3) to obtain $\tilde{z}_{k}(\tau)$. The $x$ and $y$ components of the orbit could be reconstructed by performing additional experiments with other oscillating field polarization directions [12].

A major difficulty is the loss of the complex phase of $\tilde{Z}_{k}(\omega)$. Physically, this phase indicates when an electron must leave the atom (relative to the phase of the oscillating field) in order for the trajectory to be maximally perturbed. That information is not available because the experiment averages the absorption over many cycles of the rf field.

Fortunately, in our system it is possible to recover the phase. All of the closed orbits of hydrogen in a static electric field are time-reversal symmetric about their midpoints - that is, $\tilde{z}_{k}(-\tau)=\tilde{z}_{k}(\tau)$. For orbits with this time-reversal symmetry, $\tilde{Z}_{k}$ is real, and we subsequently denote it $\tilde{Z}_{k}^{R}(\tilde{\omega})$. 
It remains only to determine this real function from its absolute value. We find its sign at $\tilde{\omega}=0$ by noting that $\tilde{Z}_{k}^{R}(0)$ is the orbit's static dipole moment, which can be found from the unscaled relation $T_{k} Z_{k}^{R}(0)=-\partial S / \partial F$. From $S=S(E, F)=\tilde{S}(\epsilon) F^{-1 / 4}$, it can be shown that

$$
\tilde{T}_{k} \tilde{Z}_{k}^{R}(0)=\frac{1}{2} \epsilon \tilde{T}_{k}+\frac{1}{4} \tilde{S}_{k} .
$$

The period $\tilde{T}_{k}$ can, in turn, be measured experimentally by varying the scaled energy and using the relation $\tilde{T}_{k}=$ $\partial \tilde{S}_{k} / \partial \epsilon$. Note that the accuracy of this measurement is not critical because we use only Eq. (4) to get the sign of $\tilde{T}_{k} \tilde{Z}_{k}^{R}$ at $\tilde{\omega}=0$.

Furthermore, since $\tilde{Z}_{k}^{R}(\tilde{\omega})$ comes from the finite-time Fourier transform of a continuous function, it depends continuously and smoothly on $\tilde{\omega}$. Therefore, knowing the sign of $\tilde{Z}_{k}^{R}$ at $\tilde{\omega}=0$, we can determine its sign for increasing $\tilde{\omega}$ by inverting the sign at each zero crossing. [Such zero crossings can be seen in Fig. 3 (below), near $\tilde{\omega}=1.65$.]

Thus $\tilde{Z}_{k}^{R}(\tilde{\omega})$, including its sign, is determined. We invert Eq. (3) by expressing $\tilde{z}_{k}(\tau)$ as a sum of smooth basis functions, $\sum_{n} a_{n} \phi_{n}(\tau)$. Then $\tilde{Z}_{k}^{R}(\tilde{\omega})=\sum_{n} a_{n} \Phi_{n}(\tau)$, where the basis functions $\Phi_{n}(\tilde{\omega})$ and $\phi_{n}(\tau)$ are related by a Fourier transform. We determine the coefficients $a_{n}$ from a least squares fit of $\tilde{Z}_{k}^{R}(\tilde{\omega})$ to the signed experimental data.

We have studied two closed orbits of lithium, the " $2 / 3$ " and the " $3 / 4$ " orbits. (The orbit label, described in [13], identifies the bifurcation in which it was created from the primitive closed orbit that exists along the positive $z$ axis.) The exact classical orbits, computed numerically, are shown in Fig. 2. The chosen energy was $\epsilon=-2.05$, slightly below the saddle point of the potential surface at $\epsilon=-2$. The orbits are both directed "downhill" toward the saddle point. They were chosen because their relatively long periods enabled the limited frequency

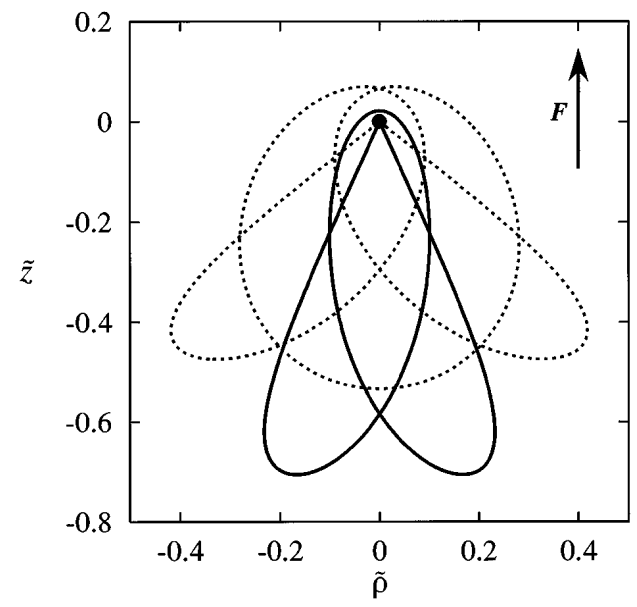

FIG. 2. Computed classical trajectories at $\epsilon=-2.05$. Solid line: $2 / 3$ orbit; dotted line: $3 / 4$ orbit. The vertical axis is the distance along the $\tilde{z}$ axis; horizontal axis is the radial distance $\tilde{\rho}$ from the $\tilde{z}$ axis. range of the experiment to access a significant fraction of the total Fourier power within $\tilde{Z}_{k}(\tilde{\omega})$.

Figure 1 shows the recurrences corresponding to these orbits at a single value of $\tilde{\omega}$ and a range of values of $\tilde{f}$. Such series of measurements were made for 17 different scaled frequencies in the range $0.6 \leq \tilde{\omega} \leq$ 4.0. The resulting values of $\tilde{T}_{k}\left|\tilde{Z}_{k}\right|$ for the $2 / 3$ and $3 / 4$ orbits are shown in Fig. 3. It can be seen that, across the experimentally accessible frequency range, agreement between data and theory is good-within about $10 \%$.

The periods for the orbits were found to be $\tilde{T}_{2 / 3}=$ $3.75 \pm 0.31$ and $\tilde{T}_{3 / 4}=4.4 \pm 0.3$ (the true values are 3.720 and 3.915 , respectively). The accuracy is limited by the calibration of the electric field. The uncertainty does not include possible systematic effects due to other orbits with similar actions, though these may be present in the $3 / 4$ orbit. Inserting these numbers into Eq. (4) correctly indicates that $\tilde{Z}^{R}(0)$ is negative for both orbits.

Figure 4 shows $\tilde{z}(\tau)$ for the two trajectories. The heavy solid lines show the orbits as reconstructed by the experiment. The qualitative behavior of the trajectories can easily be discerned. The light lines are the exact classical trajectories. Note that, as seen in Fig. 2, both orbits initially move from the nucleus in the $-z$ direction before they are turned back toward the nucleus by the electric field. The $2 / 3$ orbit loops back to the nucleus once before closing while the $3 / 4$ orbit loops back twice.

The time resolution of the reconstructed trajectories is limited by the experimental frequency range. This in turn was limited by the difficulties in coupling the rf power into the field plates, permitting an actual frequency range of $200-1260 \mathrm{MHz}$, and a scaled frequency range $0.6 \leq \tilde{\omega} \leq 4.0$. Therefore, details with a time scale shorter than $\Delta \tau \sim 1 / \tilde{\omega}_{\max }=0.25$ are not probed by this experiment. Expressed another way, at $\tilde{\omega}=4$, the oscillating field goes through about 2.5 cycles during the time of an orbit - adequate to determine only three or

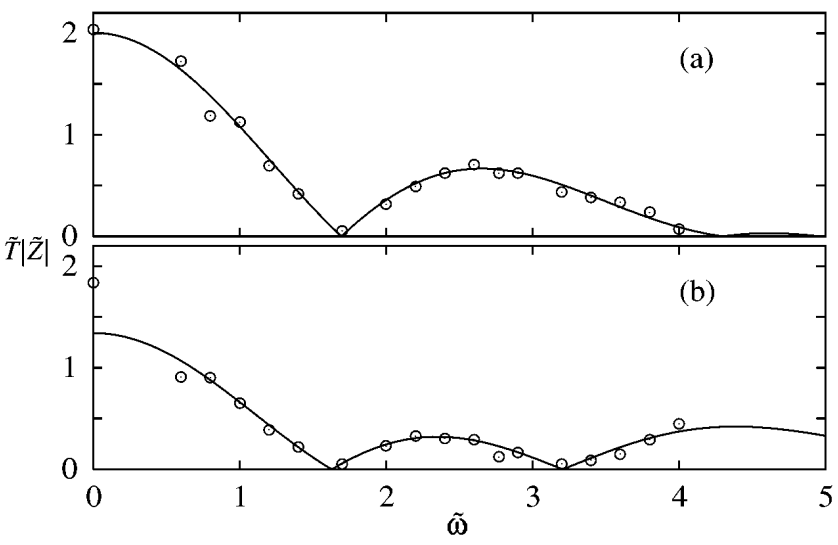

FIG. 3. Experimental measurement of $\tilde{T}|\tilde{Z}(\tilde{\omega})|$. The circles are the results of fits to data like those in Fig. 1 to the form of Eq. (2). The solid lines are classical calculation. (a) $2 / 3$ orbit; (b) $3 / 4$ orbit. The points at $\tilde{\omega}=0$ are found using Eq. (4). 


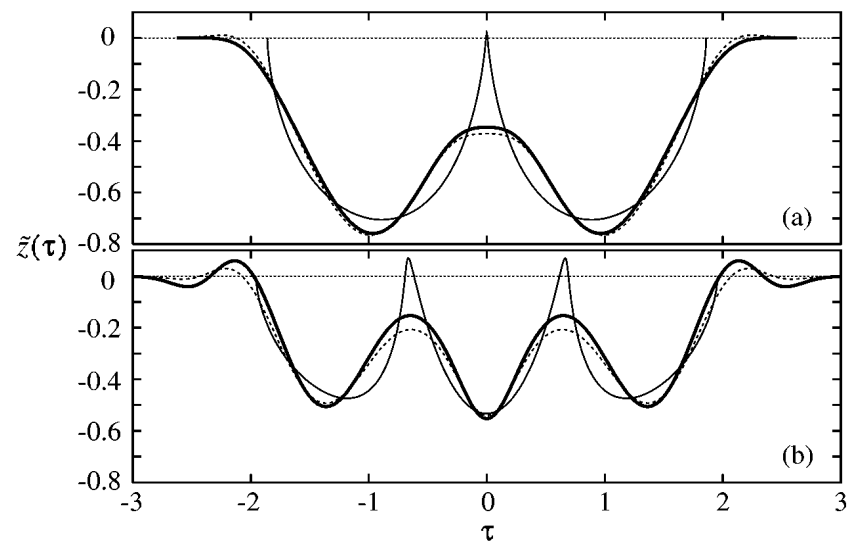

FIG. 4. Reconstruction of classical orbits. The light solid lines show the exact classical trajectories, $\tilde{z}(\tau)$. The heavy solid lines show the experimental reconstructions. The dashed lines show the exact trajectories, filtered through the experimental frequency window, $0.6 \leq \tilde{\omega} \leq 4.0$. (a) $2 / 3$ orbit; (b) $3 / 4$ orbit.

four coefficients in the orbit reconstruction. In order to illustrate the severe effect of the finite frequency range, Fig. 4 also shows the exact trajectories filtered through the experimental frequency window (dashed lines).

Our experiment produces accurate, albeit lowresolution, pictures of classical trajectories important to the Stark spectrum of lithium. Even the low-resolution reconstructions afforded by the experiment allow one to see the qualitative motion of each classical trajectory and, in particular, the different number of loops executed by each orbit-information that is available from no other experiment.

When classical trajectories are extracted from a quantum system, the resolution is necessarily limited by Heisenberg's uncertainty principle. How close is this experiment to that limit? Or put another way: To what extent could the resolution of the reconstructed trajectory be improved by increasing the frequency range of the experiment?

The semiclassical analysis relies on the assumptions that the $\mathrm{rf}$ frequency is much lower than the laser frequency, and that the classical orbits are large compared with the size of the atom. If either of these assumptions breaks down, then the fuzziness of the initial state would create fuzziness in the reconstructed trajectory. Considering that the size of the initial state is only a few Bohr radii, whereas the smallest distance probed in this experiment is a few thousand Bohr radii, we are clearly orders of magnitude away from the uncertainty principle limits. Hence, the resolution in the current experiment is limited by experimental, not fundamental, restrictions. Much more classical detail could be extracted by our method.

Our demonstration that it is possible to reconstruct classical trajectories from spectral data relies on only a few properties of the system: (i) The initial state occupies only a small region of space. (ii) Outside that region a semiclassical (short-wavelength) approximation is appro- priate. (iii) The time-dependent "probing" Hamiltonian is known, and has the form $F_{1} z \cos (\omega t)$. These assumptions are sufficient for the validity of Eq. (2), which allows us to infer $|\tilde{Z}(\omega)|$ from experimental measurements. In the present case, to obtain $\tilde{z}(\tilde{t})$ we also used the facts that the classical orbits are time-reversal symmetric (necessary because of the loss of phase information discussed above), and that the complete Hamiltonian is scaling (this is convenient but not essential to the method). Although we applied the method to the Stark spectrum of lithiuma system whose classical and quantum dynamics were already well understood - the method does not depend on the details of that system. It should be possible to apply the technique to systems for which the classical or even quantum behavior is not known.

The work at W\&M is supported by NSF Grant No. PHY-9630372 and ONR Grant No. N0001494-1-0930, and the work at MIT by NSF Grant No. PHY-9221489 and ONR Grant No. N00014-96-10484. M. R. H. acknowledges financial support from the Gabriella and Paul Rosenbaum Foundation, and thanks the Isaac Newton Institute for its hospitality. N.S. acknowledges financial support from ONR.

*Current address: Department of Physics, Harvard University, Cambridge, MA 02138.

[1] M.C. Gutzwiller, Chaos in Classical and Quantum Mechanics (Springer-Verlag, Berlin, 1990).

[2] Inverse scattering methods and the Rydberg-Klein-Rees method use scattering data or eigenvalues to reconstruct a potential energy, from which one can compute classical trajectories. See R. Newton, Scattering Theory (McGrawHill, New York, 1966), pp. 140 and 610; G. Herzberg, Spectra of Diatomic Molecules (Van Nostrand Reinhold, New York, 1950), p. 102.

[3] A. Dvornik, B.Sc. thesis, Massachusetts Institute of Technology, 1998; M. Baranger, A. Dvornik, and D. Provost (to be published).

[4] E. J. Heller, Phys. Rev. Lett. 53, 1515 (1984).

[5] M. L. Du and J. B. Delos, Phys. Rev. A 38, 1896 (1988).

[6] E. B. Bogomol'nyi, Zh. Eksp. Teor. Fiz. 96, 487 (1989) [Sov. Phys. JETP 69, 275 (1989)].

[7] A. Holle, J. Main, G. Wiebusch, H. Rottke, and K. H. Welge, Phys. Rev. Lett. 61, 161 (1988).

[8] G. Raithel, M. Fauth, and H. Walther, Phys. Rev. A 47, 419 (1993).

[9] N. Spellmeyer, D. Kleppner, M. R. Haggerty, V. Kondratovich, J. B. Delos, and J. Gao, Phys. Rev. Lett. 79, 1650 (1997).

[10] M. Courtney, N. Spellmeyer, H. Jiao, and D. Kleppner, Phys. Rev. A 51, 3604 (1995).

[11] M. R. Haggerty et al. (to be published).

[12] Because of cylindrical symmetry, Eq. (2) would be modified for the other rf field polarizations.

[13] J. Gao and J. B. Delos, Phys. Rev. A 49, 869 (1994). 